\title{
New volumetric capnography-derived parameter: a potentially valuable tool for detecting hyperventilation during cardiopulmonary resuscitation in a porcine model
}

\author{
Lili Zhang ${ }^{1 \wedge}$, Xianquan Liang ${ }^{1}$, Huadong Zhu ${ }^{2}$, Lu Yin ${ }^{2}$, Jiayuan Dai ${ }^{2}$, Danyu Liu ${ }^{2}$, Shanshan Yu ${ }^{1}$, \\ Yangyang $\mathrm{Fu}^{2}$, Kui Jin ${ }^{2}$, Jun $\mathrm{Xu}^{2} \wedge$, Xuezhong $\mathrm{Yu}^{2}$ \\ ${ }^{1}$ The Second People's Hospital of Guiyang, Guiyang, China; ${ }^{2}$ Emergency Department, Peking Union Medical College Hospital, Chinese Academy \\ of Medical Sciences, Beijing, China \\ Contributions: (I) Conception and design: L Zhang, X Liang, J Xu; (II) Administrative support: H Zhu, J Xu, X Yu; (III) Provision of study materials \\ or patients: J Xu, X Yu; (IV) Collection and assembly of data: L Zhang, X Liang; (V) Data analysis and interpretation: L Yin, J Dai, D Liu, S Yu, Y \\ Fu, K Jin; (VI) Manuscript writing: All authors; (VII) Final approval of the manuscript: All authors. \\ Correspondence to: Jun Xu. Emergency Department, Peking Union Medical College Hospital, 1 Shuaifuyuan Wangfujing, Dongcheng District, \\ Beijing 100730, China. Email: xujunfree@126.com; Xuezhong Yu. Emergency Department, Peking Union Medical College Hospital, 1 Shuaifuyuan \\ Wangfujing, Dongcheng District, Beijing 100730, China. Email: yxzpumch@126.com.
}

Background: Volumetric capnography is increasingly being applied in cardiopulmonary resuscitation. However, during cardiopulmonary resuscitation, the abnormal ventilation state affects the monitoring effect of the most commonly used capnography-derived parameter, the partial carbon dioxide end-tidal pressure $\left(\mathrm{PetCO}_{2}\right)$. In this study, we evaluated the ability of a new volumetric capnography-derived parameter, the ratio between the $\mathrm{PetCO}_{2}$ and the volume of carbon dioxide $\left(\mathrm{CO}_{2}\right)$ eliminated per min and per kilogram of body weight, for detecting hyperventilation during cardiopulmonary resuscitation.

Methods: We used 12 porcine models of primary ventricular fibrillation-induced cardiac arrest. Ventricular fibrillation was induced and left untreated for $4 \mathrm{~min}$. Standardized chest compressions were performed throughout the experiment using mechanical cardiopulmonary resuscitation. Following 5 min of normal ventilation as a washout period, each animal underwent 4 types of ventilation. The main outcome measures were the $\mathrm{PetCO}_{2}$, the ratio between the $\mathrm{PetCO}_{2}$ and the volume of $\mathrm{CO}_{2}$ eliminated per min and per kilogram of body weight with each ventilation type.

Results: Different ventilation types had a significant effect on the volumetric capnography-derived parameters. The PetCO $\mathrm{O}_{2}$ and ratio between the $\mathrm{PetCO}_{2}$ and the volume of $\mathrm{CO}_{2}$ eliminated per min and per kilogram of body weight values during cardiopulmonary resuscitation was significantly higher in non-hyperventilating than in hyperventilating animals. The ratio reflected hyperventilation accurately and immediately, with an area under the curve (AUC) of 0.98 . The optimal cut-off point of the ratio for discriminating hyperventilation from non-hyperventilation was 6.36, with a sensitivity and specificity of 0.99 and 0.89 , respectively.

Conclusions: The ratio between the $\mathrm{PetCO}_{2}$ and the volume of $\mathrm{CO}_{2}$ eliminated per min and per kilogram of body weight showed good performance in discriminating hyperventilation from non-hyperventilation and was sensitive to changes in ventilation status. This ratio may be a valuable clinical indicator for monitoring the ventilation status during cardiopulmonary resuscitation.

Keywords: Cardiac arrest; cardiopulmonary resuscitation (CPR); end-tidal $\mathrm{CO}_{2}$ partial pressure $\left(\mathrm{PetCO}_{2}\right)$; hyperventilation

^ ORCID: Lili Zhang, 0000-0002-0012-6514; Jun Xu, 0000-0001-5014-4238. 
Submitted Jan 08, 2021. Accepted for publication Apr 22, 2021.

doi: $10.21037 /$ jtd-21-50

View this article at: http://dx.doi.org/10.21037/jtd-21-50

\section{Introduction}

Capnography is now recommended as the standard monitoring method for advanced cardiopulmonary resuscitation (CPR) $(1,2)$. The advantages of capnography include assessment of the correct placement of the endotracheal tube (3), evaluating the quality of chest compressions (CC) $(4,5)$, monitoring the early return of spontaneous circulation (6), and predicting the patient prognosis $(1,7,8)$. Additionally, capnography is recommended to monitor ventilation rates and avoid hyperventilation during CPR, according to current guidelines (7). Hyperventilation may be caused by an excessive respiratory rate (RR), high tidal volume (VT), or a combination of both. However, capnography focuses on RR monitoring, both visually and automatically $(9,10)$.

During CPR, the purpose of ventilation is to ensure that sufficient oxygen is supplied and carbon dioxide $\left(\mathrm{CO}_{2}\right)$ is removed, thereby maintaining proper blood oxygen and $\mathrm{CO}_{2}$ levels. Simultaneously, another important principle of ventilation is to avoid adverse effects on hemodynamics (11-13). Thus, it is not reasonable to evaluate ventilation status using RR, VT, or minute volume (MV) alone. Although arterial blood gas analysis results provide information about the ventilation status, arterial blood sampling is not routinely available and does not provide real-time results. Therefore, volumetric capnography (Vcap)-derived variables can be used to develop parameters for the real-time monitoring of ventilation status during resuscitation.

Using Vcap provides continuous and noninvasive information about the fractional concentration or partial pressure of exhaled $\mathrm{CO}_{2}$ against the expired volume of 1 breath $(14,15)$. One of the most commonly used Vcapderived parameters, the end-tidal partial carbon dioxide pressure $\left(\mathrm{PetCO}_{2}\right)$, is affected by hyperventilation, as confirmed in animal experiments (16) and clinical observational studies (17-19). The $\mathrm{PetCO}_{2}$ decreases with an increase in $M V$ (16). Another Vcap-derived parameter, the volume of $\mathrm{CO}_{2}$ eliminated per $\min \left(\mathrm{V}^{\prime} \mathrm{CO}_{2}\right)$, can reflect metabolic $\mathrm{CO}_{2}$ production, provided that ventilation and perfusion conditions remain unchanged (15). According to the results of our previous animal experiments (20), the accumulated and produced $\mathrm{CO}_{2}$ is eliminated quickly by hyperventilation, as manifested by a rapid increase in $\mathrm{V}^{\prime} \mathrm{CO}_{2}$ within 2-3 min after the change in ventilation status. The $\mathrm{V}^{\prime} \mathrm{CO}_{2}$ then gradually decreases to a stable level, reflecting the true metabolic status of the body. When analyzing such experimental results, an individual's body weight should be considered. A modified parameter, the volume of $\mathrm{CO}_{2}$ eliminated per min per kilogram of body weight $\left(\mathrm{V}^{\prime} \mathrm{CO}_{2} / \mathrm{kg}\right)$, has been used for this analysis and has provided more valuable conclusions. It is theoretically feasible to use the ratio between the 2 indices to determine the ventilation status. However, this parameter's ability has not been studied in terms of its ability to detect hyperventilation to date.

By reviewing previously collected data, we aimed to construct an algorithm to monitor animals' ventilation status during resuscitation. We hypothesized that $\mathrm{PetCO}_{2}$ would decrease and $\mathrm{V}^{\prime} \mathrm{CO}_{2} / \mathrm{kg}$ would increase after hyperventilation began, after which the $\mathrm{PetCO}_{2} /\left(\mathrm{V}^{\prime} \mathrm{CO}_{2} /\right.$ $\mathrm{kg}$ ) (PV ratio) would decrease significantly.

We present the following article in accordance with the ARRIVE reporting checklist (available at http://dx.doi. org/10.21037/jtd-21-50).

\section{Methods}

Data from a randomized crossover study were retrospectively analyzed. All animals had complete laboratory records, including hemodynamic and respiratory parameters. All animals received humane care following the principles of laboratory animal care formulated by the Ministry of Science and Technology of the People's Republic of China. The Ethics Committee for Animal Experiments at Peking Union Medical College Hospital approved the experimental protocol. The pigs were kept in animal houses with metal fences and concrete floors, and 2 pigs shared 1 cage with an average area of $1.5-3 \mathrm{~m}^{2}$ per pig. The environment was well ventilated. The ambient temperature was controlled at $18-25^{\circ} \mathrm{C}$, and the relative humidity was $30-50 \%$. Pigs were fed with a special feed twice a day. The managers document daily observations of health, status, food, and water of the pigs. A total of 12 male domestic pigs aged 3-4 months and weighing $35.6 \pm 2.0 \mathrm{~kg}$ 


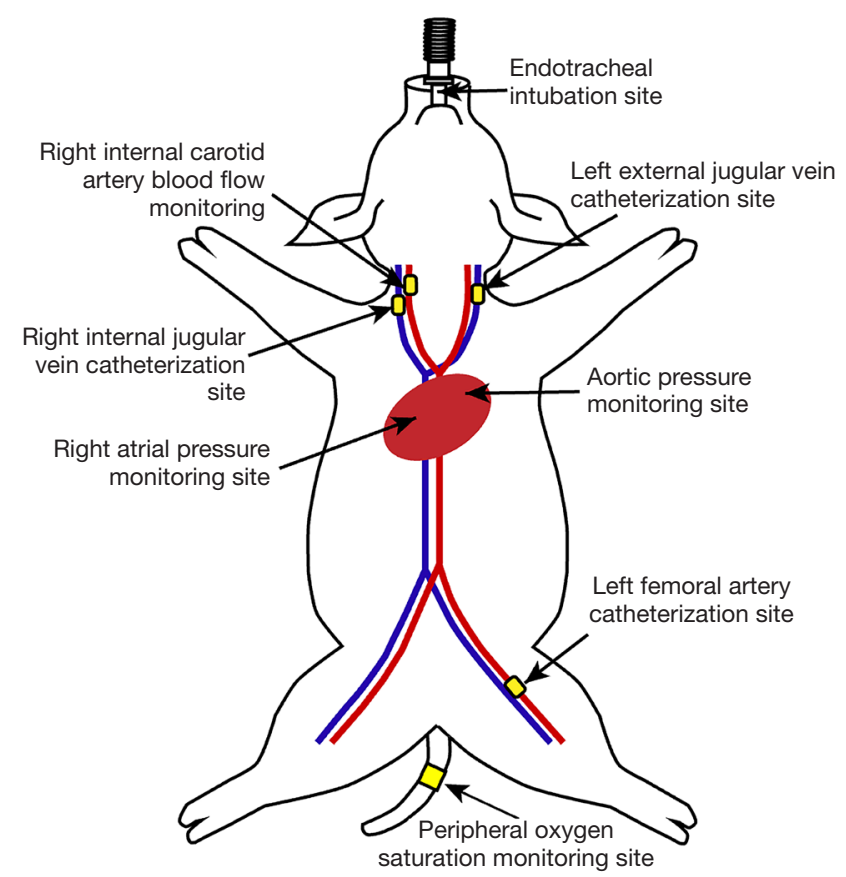

Figure 1 An electronic-tipped catheter (Mikro-Tip Transducer, Millar Instruments, Houston, TX, USA) was inserted via the left femoral artery for continuous monitoring of the aortic pressure. The left external jugular vein was surgically exposed and cannulated with an 8-French introducer sheath to monitor RAP. The RAP was maintained at 3-8 $\mathrm{mmHg}$ during the preparation phase. Carotid blood flow was measured using an ultrasonic flow probe $(6 \mathrm{~mm})$ surrounding the internal carotid artery (Transonic Systems, Ithaca, NY, USA). The right internal jugular vein was also fitted with a catheter to provide access for ventricular fibrillation induction. RAP, right atrial pressure.

were studied.

\section{Animal preparation}

Animal preparation procedures, including anesthesia, fixation, mechanical ventilation, and instrumentation methodology, were undertaken as previously described (20). The catheter position, pressure probe, and flow probe placement are shown in Figure 1. The animals were mechanically ventilated with a SynoVent 800 respirator in the baseline data collection stage (Mindray Biological Medical Electronic Co., Ltd., Shenzhen, Guangdong, China). The respirator was started in volume-controlled ventilation (VCV) with ventilator settings as follows: VT of $10 \mathrm{~mL} / \mathrm{kg}$; positive end-expiratory pressure (PEEP) of
$4 \mathrm{cmH}_{2} \mathrm{O}$; inspiratory to expiratory time ratio (I:E) of 1:1.5-2. The RR was titrated to maintain $\mathrm{PetCO}_{2}$ of 38$42 \mathrm{mmHg}$ and pulse oxygen saturation $\left(\mathrm{SPO}_{2}\right)$ at $>95 \%$. After the preparation period, the animals were stabilized for $15 \mathrm{~min}$ under the following conditions: heart rate (HR) of $80-100$ beats/min, $\mathrm{PetCO}_{2}$ of $38-42 \mathrm{mmHg}, \mathrm{SPO}_{2}$ $>95 \%$, right atrial pressure (RAP) of $3-8 \mathrm{mmHg}$, and body temperature of $37.0-38.0^{\circ} \mathrm{C}$. The animals then received a $0.6 \mathrm{mg} / \mathrm{kg}$ intravenous bolus of rocuronium bromide (NV Organon, Oss, Netherlands). To inhibit gasping, rocuronium bromide continued to be infused at a rate of 0.1-0.2 $\mathrm{mg} / \mathrm{kg} / \mathrm{h}$ during CPR.

\section{Experimental protocol}

Clinical research has reported that rescue personnel's ventilation frequency may be up to 30 breaths/min during out-of-hospital cardiac arrest (CA) rescue (10). This result prompted us to investigate 3 levels of RR $(10,20$, and 30 breaths/min). Simultaneously, we also wanted to compare the effect of hyperventilation caused by high VT on the target parameters. Since a high VT may cause lung injury, and as exceeding double the normal VT is not common clinically, we studied only 2 VT levels (10 and $20 \mathrm{~mL} / \mathrm{kg}$ ). Therefore, this study investigated the following 4 ventilation types: normal ventilation (NV), RR at 10 breaths/min, VT at $10 \mathrm{~mL} / \mathrm{kg}$; double RR hyperventilation (DRRH), RR at 20 breaths/min, VT at $10 \mathrm{~mL} / \mathrm{kg}$, double VT hyperventilation (DVTH), RR at 10 breaths $/ \mathrm{min}, \mathrm{VT}$ at $20 \mathrm{~mL} / \mathrm{kg}$; triple RR hyperventilation (TRRH), RR at 30 breaths $/ \mathrm{min}$, and VT at $10 \mathrm{~mL} / \mathrm{kg}$. According to the ventilation type used, the 12 pigs were divided into 4 groups ( $\mathrm{n}=3$ per group).

Each animal underwent 2 successive sessions of the original experiment. In this retrospective analysis, we only included data from the first session for analysis. The experimental timeline of the first session and grouping is shown in Figure 2. In the first session of the experiment, no vasoactive drugs, including epinephrine or electric defibrillation, were used. Methods of CA induction and experimental processes were used as described previously (20). Briefly, ventricular fibrillation (VF) was induced after recording the baseline parameters. The animals were then disconnected from the ventilator support, and drug infusion was halted. After 4 min of untreated VF, CCs were started with an impedance threshold device (ITD) with a frequency of 120 compressions/min at a depth of $25 \%$ of the anteroposterior chest diameter, using special 


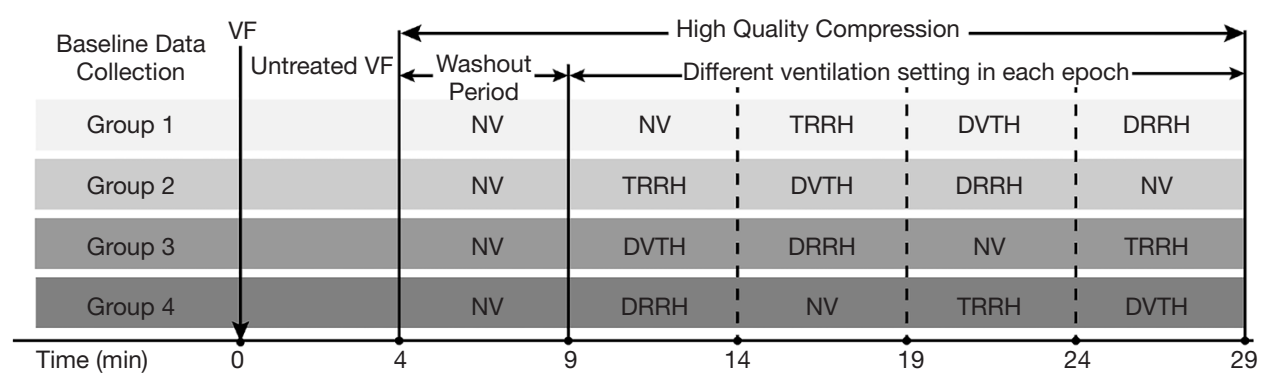

Figure 2 Experimental timeline. RR, respiratory rate; VT, tidal volume; VF, ventricular fibrillation; NV, normal ventilation (RR: 10 breaths/min and VT: $10 \mathrm{~mL} / \mathrm{kg}$ ); DRRH, double RR hyperventilation (RR: 20 breaths/min and VT: $10 \mathrm{~mL} / \mathrm{kg}$ ); DVTH, double VT hyperventilation (RR: 10 breaths/min and VT: $20 \mathrm{~mL} / \mathrm{kg}$ ); TRRH, triple RR hyperventilation (RR: $30 \mathrm{breaths} / \mathrm{min}$ and VT: $10 \mathrm{~mL} / \mathrm{kg}$ ).

animal mechanical CPR equipment (WISH-SL-FS-A, Wuhan, Hubei, China). The same compression was applied throughout the experimental process without interruption. Ventilation was synchronized with the mechanical CC. The percentage of time being compressed was $40 \%$ of the entire compression cycle, and the percentage of time being held was $20 \%$. The VCV was selected with ventilator settings as follows: trigger was disabled, a fraction of inspiratory oxygen of $100 \%$, PEEP of $0 \mathrm{cmH}_{2} \mathrm{O}$, I:E of $1: 1.5-2$, and either VT or RR, depending on the group allocation. To achieve stable $\mathrm{CO}_{2}$ excretion, a 5 min washout period was set to avoid interference of $\mathrm{CO}_{2}$ retention during CA. Following the 5 min standard CPR with $\mathrm{NV}$, each animal underwent 4 ventilation trials of $5 \mathrm{~min}$, based on a randomized crossover design. The order of ventilation types was counterbalanced and randomized using the closed envelope method. The animals were euthanized when the experiment had been completed.

\section{Measurements}

A T8 Mindray monitor (Mindray Biological Medical Electronic Co., Ltd., Shenzhen, Guangdong, China) was used to monitor the following parameters continuously: HR, electrocardiogram, RAP, central aortic pressure (AoP), and $\mathrm{SPO}_{2}$. Coronary perfusion pressure (CPP) was calculated as the difference between the AoP and RAP during the CPR decompression phase. Continuous acquisition of graphical on-screen hemodynamic data, including AoP, RAP, CPP, and carotid blood flow, was performed using a multichannel physiological recorder (BIOPAC MP150, BIOPAC Systems, Inc., Goleta, CA, USA). The RR, VT, and MV were recorded continuously using the respiratory background storage system.
This experiment mainly studied 2 Vcap-derived parameters, namely, $\mathrm{PetCO}$ and $\mathrm{V}^{\prime} \mathrm{CO}_{2} / \mathrm{kg}$. The measurements of $\mathrm{PetCO}_{2}$ and $\mathrm{V}^{\prime} \mathrm{CO}_{2}$ were obtained directly from a Vcap connector (NICO; Respironics Inc., Wallingford, CT, USA). $\mathrm{V}^{\prime} \mathrm{CO}_{2} / \mathrm{kg}$ was calculated by dividing $\mathrm{V}^{\prime} \mathrm{CO}_{2}$ by the animal's body weight in the data processing stage after the experiment. The $\mathrm{PV}$ ratio was calculated by dividing $\mathrm{PetCO}_{2}$ by $\mathrm{V}^{\prime} \mathrm{CO}_{2} / \mathrm{kg}$. The accuracy of the $\mathrm{V}^{\prime} \mathrm{CO}_{2}$ calculation was affected by the oscillation of the gas in the ventilation tube during CPR. We resolved this problem using an ITD since its design principle allows the exhaled gas to be discharged in the compression phase and prevents the gas from entering the decompression phase (17). The reasons for this are explained in detail elsewhere (20).

All of the parameters mentioned above were measured at $30 \mathrm{~s}$ intervals, and the mean values were used in the analysis.

\section{Statistical analysis}

All statistical analyses were performed using the statistical software SPSS version 25.0 (IBM Corp., Armonk, NY, USA). Normally distributed data were expressed as mean \pm standard deviation (SD), and repeated-measures analysis of variance (RM-ANOVA) was used for comparisons. Non-normally distributed variables were expressed as medians (upper quartile, lower quartile), and the Friedman test was used for comparison. A generalized linear mixed model was used to test the changes in hemodynamic and ventilatory parameters between ventilation types. A pairwise comparison with Bonferroni adjustment was performed for post-hoc analysis. Receiver operating characteristic (ROC) curves with area under the curve (AUC) analyses were used to assess the ability of the PV ratio to discriminate between 


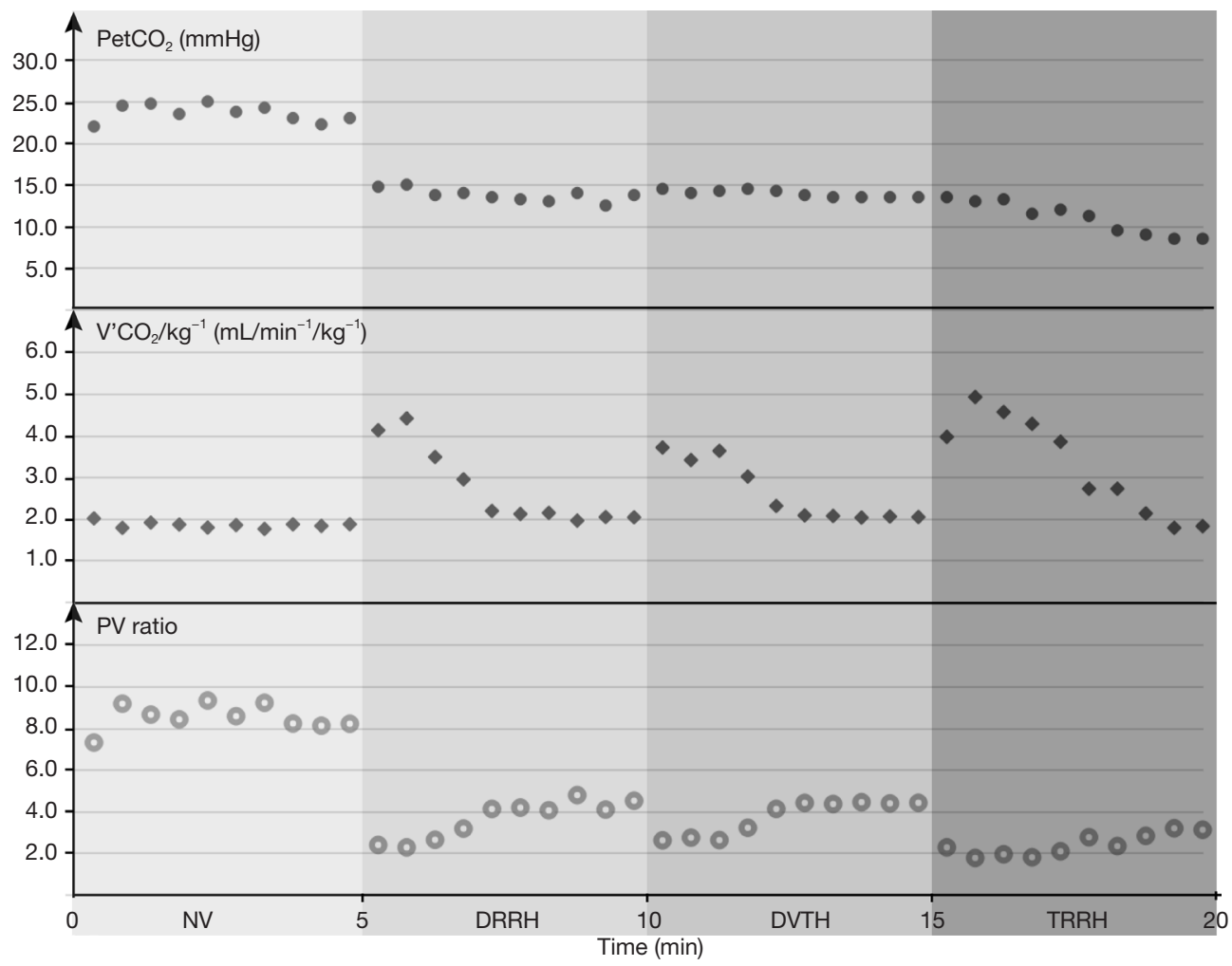

Figure $3 \mathrm{PetCO}_{2}, \mathrm{~V}^{\prime} \mathrm{CO}_{2} / \mathrm{kg}$, and $\mathrm{PV}$ ratio for each ventilation type during CPR. PV ratio, the ratio between $\mathrm{PetCO}_{2}$ and $\mathrm{V}^{\prime} \mathrm{CO}_{2} / \mathrm{kg}$. PetCO $\mathrm{C}_{2}$, partial end-tidal carbon dioxide pressure; $\mathrm{V}^{\prime} \mathrm{CO}_{2} / \mathrm{kg}$, the volume of carbon dioxide eliminated per min and per kilogram of body weight; RR, respiratory rate; VT, tidal volume; VF, ventricular fibrillation; NV, normal ventilation (RR: 10 breaths/min and VT: $10 \mathrm{~mL} / \mathrm{kg}$ ); DRRH, double RR hyperventilation (RR: 20 breaths/min and VT: $10 \mathrm{~mL} / \mathrm{kg}$ ); DVTH, double VT hyperventilation (RR: 10 breaths/min and VT: $20 \mathrm{~mL} / \mathrm{kg}$ ); TRRH, triple RR hyperventilation (RR: 30 breaths/min and VT: $10 \mathrm{~mL} / \mathrm{kg}$ ).

hyperventilation and non-hyperventilation conditions and between 2-fold (DRRH, DVTH) and 3-fold normal MV (TRRH). Statistical significance was considered when twotailed $\mathrm{P}$ values $<0.05$.

\section{Ethical statement}

The Ethics Committee for Animal Experiments at the Peking Union Medical College Hospital approved the experimental protocol (XHDW-2019-023). All animals received humane care in accordance with the "principles of laboratory animal care" formulated by the Ministry of Science and Technology of the People's Republic of China.

\section{Results}

Data from 12 pigs were analyzed, and each pig underwent 4 types of ventilation; thus, a total of 48 data epochs were used for analysis.

A single animal from each group was randomly selected to evaluate the change in Vcap-derived parameters during the 4 ventilation types, as shown in Figure 3. After changing the ventilation type, $\mathrm{PetCO}_{2}$ decreased rapidly and then quickly reached a stable state; $\mathrm{V}^{\prime} \mathrm{CO}_{2} / \mathrm{kg}$ first increased and then decreased, gradually reaching a stable state within 23 min. The PV ratio decreased rapidly at the beginning of the switch to hyperventilation and then gradually recovered to a stable state after $1-2 \mathrm{~min}$.

The generalized linear mixed model revealed that the 4 different ventilation types had no significant effect on hemodynamic indices, but a significant effect was noted on Vcap-derived parameters $(\mathrm{P}<0.001)$. The $\mathrm{PetCO}_{2}$, $\mathrm{V}^{\prime} \mathrm{CO}_{2} / \mathrm{kg}$, and the $\mathrm{PV}$ ratio during each ventilation type are displayed in Figure 4. The average values of the hemodynamic variables and Vcap-derived parameters are listed in Table 1. When comparing the 4 ventilation 

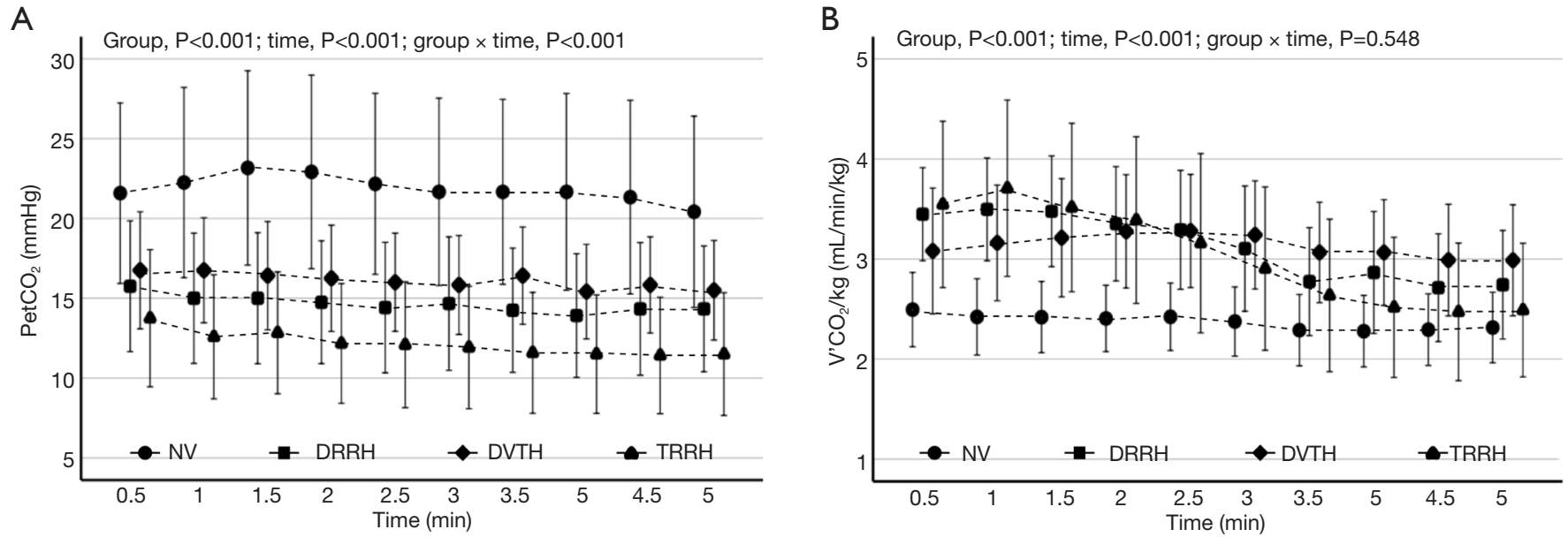

C

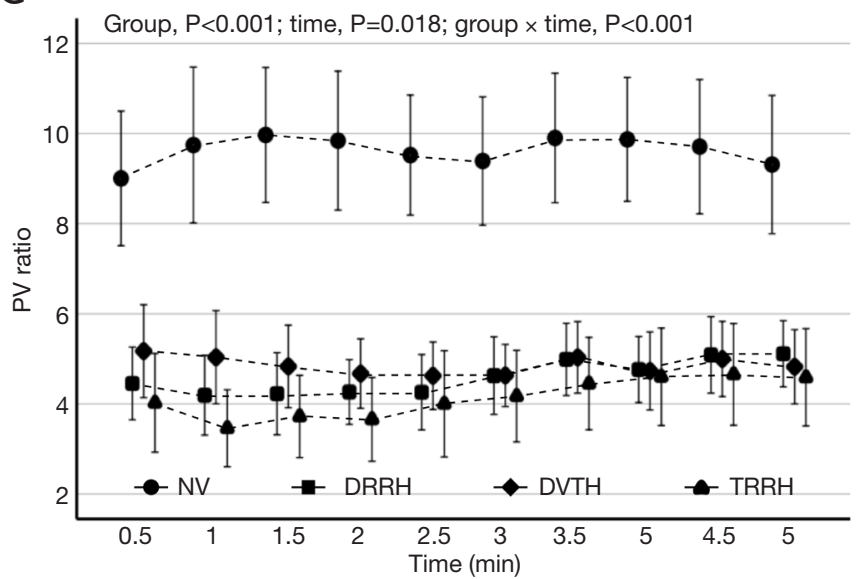

Figure $4 \mathrm{PetCO}_{2}, \mathrm{~V}^{\prime} \mathrm{CO}_{2} / \mathrm{kg}$, and $\mathrm{PV}$ ratio during each ventilation type. (A) $\mathrm{PetCO}_{2}$ during each ventilation type. (B) $\mathrm{V}^{\prime} \mathrm{CO}_{2} / \mathrm{kg}$ during each ventilation type. (C) PV ratio during each ventilation type. RR, respiratory rate; VT, tidal volume; PetCO ${ }_{2}$, the partial pressure of end-tidal carbon dioxide; $\mathrm{V}^{\prime} \mathrm{CO}_{2} / \mathrm{kg}$, the volume of carbon dioxide eliminated per min and per kilogram of body weight; $\mathrm{PV}$ ratio, the ratio of PetCO $/$ $\left(\mathrm{V}^{\prime} \mathrm{CO}_{2} / \mathrm{kg}\right.$ ); NV, normal ventilation (RR: 10 breaths/min and VT: $10 \mathrm{~mL} / \mathrm{kg}$ ); DRRH, double RR hyperventilation (RR: 20 breaths/min and VT: $10 \mathrm{~mL} / \mathrm{kg}$ ); DVTH, double VT hyperventilation (RR: 10 breaths/min and VT: $20 \mathrm{~mL} / \mathrm{kg}$ ); TRRH, triple RR hyperventilation (RR: 30 breaths/min and VT: $10 \mathrm{~mL} / \mathrm{kg}$ ).

types' analyzed parameters, the RM-ANOVA of PetCO and $\mathrm{PV}$ ratio revealed significant group effects, time effects, and group-time interactions. The RM-ANOVA of $\mathrm{V}^{\prime} \mathrm{CO}_{2} / \mathrm{kg}$ revealed significant time and group effects. All hemodynamic parameters decreased over time, regardless of ventilation type. A significant decrease in $\mathrm{PetCO}_{2}$ and $\mathrm{PV}$ ratio was found in the DRRH, DVTH, and TRRH groups in comparison with the $\mathrm{NV}$ group. The $\mathrm{PetCO}_{2}$ and $\mathrm{PV}$ ratios showed no significant differences between the DRRH and DVTH groups. Although the value of $\mathrm{V}^{\prime} \mathrm{CO}_{2} / \mathrm{kg}$ was the highest in the NV group, no significant difference was found among the other 3 groups.
The performance of the PV ratio for detecting hyperventilation during CRP is shown in Figure 5. For discriminating hyperventilation from non-hyperventilation, the AUC for the PV ratio was 0.98 [95\% confidence interval $(\mathrm{CI}): 0.97$ to $0.99, \mathrm{P}<0.001]$. As determined by the Youden index, the optimal cut-off point of the PV ratio for discriminating hyperventilation from non-hyperventilation was 6.36 , with a sensitivity of 0.99 , specificity of 0.89 , a positive likelihood ratio of 9.00, and a negative likelihood ratio of 0.01. However, for discriminating 2-fold (DRRH, DVTH) from 3-fold normal MV (TRRH), the AUC was 0.68 (95\% CI: 0.61 to $0.74, \mathrm{P}<0.001$ ). 
Table 1 Key hemodynamic variables and Vcap-derived parameters for the 4 ventilation types during cardiopulmonary resuscitation

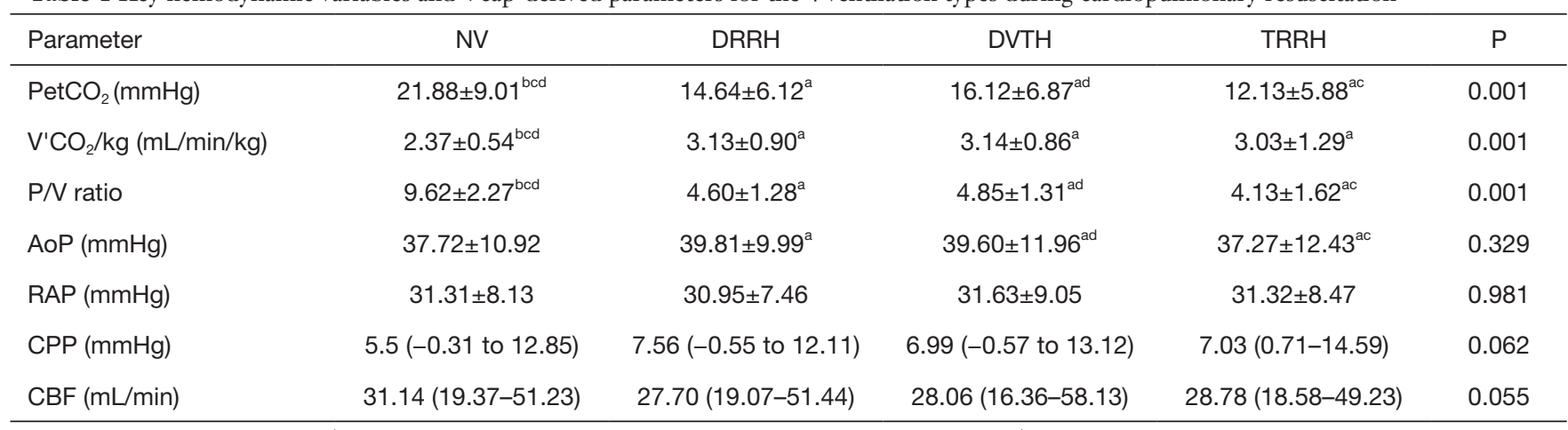

${ }^{a}, \mathrm{P}<0.05$ compared to NV; ${ }^{b}, \mathrm{P}<0.05$ compared to DRRH; ${ }^{\mathrm{c}}, \mathrm{P}<0.05$ compared to DVTH; ${ }^{\mathrm{d}}, \mathrm{P}<0.05$ compared to TRRH. Values are shown as mean \pm standard deviation or medians and interquartile ranges. $P$ et $\mathrm{CO}_{2}$, partial end-tidal carbon dioxide pressure; $\mathrm{V}^{\prime} \mathrm{CO}_{2} / \mathrm{kg}, \mathrm{volume}$ of carbon dioxide eliminated per minute and per $\mathrm{kg}$ of body weight; $\mathrm{PV}$ ratio, ratio of $\mathrm{PetCO} /\left(\mathrm{V}^{\prime} \mathrm{CO}_{2} / \mathrm{kg}\right)$; RR, respiratory rate; $\mathrm{VT}$, tidal volume; VF, ventricular fibrillation; NV, normal ventilation (RR: 10 breaths/min and VT: $10 \mathrm{~mL} / \mathrm{kg}$ ); DRRH, double RR hyperventilation (RR: 20 breaths/min and VT: $10 \mathrm{~mL} / \mathrm{kg}$ ); DVTH, double VT hyperventilation (RR: 10 breaths/min and VT: $20 \mathrm{~mL} / \mathrm{kg}$ ); TRRH, triple RR hyperventilation (RR: 30 breaths/min and VT: $10 \mathrm{~mL} / \mathrm{kg}$ ). AoP, central aortic pressure; RAP, right atrial pressure; CPP, coronary perfusion pressure; CBF, carotid blood flow.
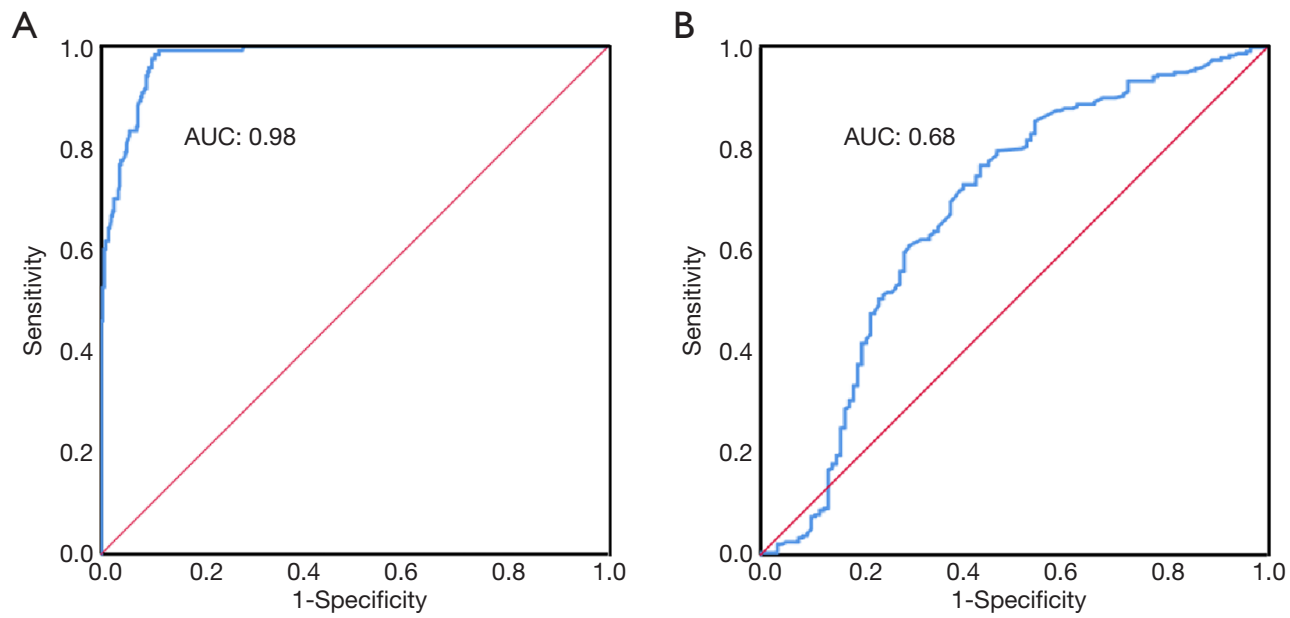

Figure 5 The performance of the PV ratio for detecting hyperventilation during CRP. (A) ROC curves evaluating the ability of the PV ratio to discriminate between hyperventilation and non-hyperventilation. AUC of panel A: 0.98 (95\% CI: 0.97 to 0.99 ). (B) ROC curves evaluating the PV ratio ability to discriminate between 2-fold normal minute volume from 3-fold normal minute volume. AUC of panel B: 0.68 (95\% CI: 0.61 to 0.74 ). $P$ etCO $\mathrm{CO}_{2}$, the partial pressure of end-tidal carbon dioxide; $\mathrm{V}^{\prime} \mathrm{CO}_{2} / \mathrm{kg}$, the volume of carbon dioxide eliminated per min and per kilogram of body weight; PV ratio, the ratio of $\mathrm{PetCO}_{2} /\left(\mathrm{V}^{\prime} \mathrm{CO}_{2} / \mathrm{kg}\right)$; ROC, receiver operating characteristic; AUC, area under the curve; CI, confidence interval.

\section{Discussion}

We found a significant decrease in the $\mathrm{PV}$ ratio when porcine CPR models were hyperventilated. Moreover, the $\mathrm{PV}$ ratio decrease was significantly faster than that of the other 2 Vcap-derived parameters. A significant decrease in the PV ratio was observed when the RR increased. In hyperventilation caused by simply increasing VT (DVTH), the PV ratio also decreased significantly compared with NV. Regarding the ability to detect hyperventilation from non-hyperventilation, the $\mathrm{PV}$ ratio showed excellent performance, with an AUC of 0.98. This parameter's optimal cut-off point was 6.36 , providing evidence that lower values during CPR are highly likely to indicate 
hyperventilation. However, the AUC for the PV ratio was only 0.68 for discriminating 2-fold (DRRH, DVTH) from 3-fold normal MV (TRRH).

These findings indicate that the PV ratio is a useful parameter for monitoring the ventilation status in real-time and noninvasively during CPR. The 2015 American Heart Association (AHA) CPR guidelines emphasize avoiding excessive ventilation to achieve high-quality CPR (21). Although the guidelines recommend that the provider should deliver 10 breaths per min when ventilating via an advanced airway, hyperventilation during advanced life support remains common among uncertified or inexperienced CPR providers (22). An inappropriately high $\mathrm{RR}$ ( $>25$ breaths/min) is commonly observed during CPR $(11,23)$. For this reason, capnography is important as a continuous monitoring method to avoid hyperventilation. By analyzing previous animal experimental data, we found that a simple calculation using Vcap-derived parameters may determine the relationship between gas supply and $\mathrm{CO}_{2}$ removal. This value facilitates making changes to the mode of ventilation monitoring during resuscitation from monitoring $\mathrm{RR}$ or $\mathrm{MV}$ to evaluate the correspondence between the $\mathrm{MV}$ and $\mathrm{CO}_{2}$ production.

The $\mathrm{PetCO}_{2}$ is determined by $\mathrm{CO}_{2}$ production, pulmonary blood flow, and alveolar ventilation (24). In our experiment, a unified CA animal model was used, and the same quality $\mathrm{CC}$ was provided to ensure relative stability of $\mathrm{CO}_{2}$ production and pulmonary blood flow during $\mathrm{CPR}$. A 2- or 3-fold MV was then set by adjusting the RR or VT separately to evaluate the effect of different levels of hyperventilation on $\mathrm{PetCO}_{2}$. Similar to other experiments, we found that with the increase in $\mathrm{MV}, \mathrm{PetCO}_{2}$ decreased gradually, and its monitoring value for the quality of CC and prognosis decreased. The $\mathrm{PetCO}_{2}$ decreased to a stable level within 1-2 min after switching to hyperventilation modes.

The other indicator, $\mathrm{V}^{\prime} \mathrm{CO}_{2} / \mathrm{kg}$, first showed an increasing trend and then decreased to a stable state, which took approximately $2-3 \mathrm{~min}$. It was previously found that $\mathrm{V}^{\prime} \mathrm{CO}_{2} / \mathrm{kg}$ showed no significant difference between NV and hyperventilation caused by increased RR after reaching a stable state and that it could be used as a more stable indicator to determine the quality of $\mathrm{CC}$ during hyperventilation. At the same time, $\mathrm{V}^{\prime} \mathrm{CO}_{2} / \mathrm{kg}$ performed better than $\mathrm{PetCO}_{2}$ in predicting survival. According to the change in the trend of $\mathrm{V}^{\prime} \mathrm{CO}_{2} / \mathrm{kg}$ provided by our experimental animal data, once hyperventilation occurs, the parameter will suddenly increase to become "normal gradually", and it loses the ability to identify hyperventilation after $3 \mathrm{~min}$. Therefore, for this indicator, in the actual clinical CPR scenario, the change in trend in the process of CPR is more valuable than the observation value at a given point. In our study, $\mathrm{PetCO}_{2}$ decreased, and $\mathrm{V}^{\prime} \mathrm{CO}_{2} / \mathrm{kg}$ increased immediately after hyperventilation. This makes the PV ratio a sensitive index for detecting ventilation changes rapidly. The results showed that the PV ratio decreased rapidly and significantly, then gradually and slightly increased, and finally stabilized at a lower level after approximately $2 \mathrm{~min}$. Based on the advantages of the PV ratio mentioned above, it may be another valuable index for detecting hyperventilation during CPR.

Another meaningful property of the PV ratio is that it reflects the essence of ventilation, meaning whether the volume of gas provided matches the volume required for $\mathrm{CO}_{2}$ removal. According to the current guidelines, we standardized the ventilation settings for animals, ignoring individual differences. In real-world CPR, the ventilation needs of patients with CA are often complex and difficult to assess. In patients with lung diseases, such as acute respiratory distress syndrome, the dead space is enlarged, leading to decreased effective alveolar ventilation. Under these circumstances, if ventilation support is still implemented according to the guidelines, there is a risk of insufficient ventilation. Therefore, monitoring ventilation quality during CPR should not be limited to monitoring the RR or MV, but instead should be based on the volume supplied to meet the demand for $\mathrm{CO}_{2}$ removal. The PV ratio, a new Vcap-derived parameter, can be obtained through simple calculations and shows a good ability to detect hyperventilation and changes immediately after hyperventilation in an animal model. Our results were based on animal experiments, and the values of the optimal cut-off point and reference ranges in the results are not suitable for clinical applications. However, this parameter points to the possibility of future noninvasive, real-time, and continuous monitoring of ventilation status in clinical CPR.

It should be noted that the definition of NV during CPR in our experiment was not accurate. The amount of gas provided by the ventilator should match the reduced pulmonary blood flow during resuscitation. The NV parameters set in the experiment may have resulted in the overventilation of the animals. Although we standardized the animal CA model as much as possible, the heterogeneity between individuals made it difficult to set a unified "NV parameter". It is difficult to monitor pulmonary blood flow in real-time. Blood gas analysis may provide some 
information, but it is usually delayed. Therefore, in the selection of NV parameters, our protocol is not perfect.

While the PV ratio may be a valuable parameter, we still face challenges in applying it to real-world resuscitation. Accurate monitoring of RR and Vcap-derived parameters relies on high-quality capnogram recordings. In future real-world clinical resuscitation scenarios, the PV ratio application will depend on the improvement of monitoring technology and equipment.

\section{Limitations}

This study had several limitations. First, this was a retrospective analysis of previously performed experiments; thus, the experimental design was not based on this study's purpose. Second, we used healthy animals, which may not represent the conditions leading to $\mathrm{CA}$, since in clinical resuscitation, most patients with $\mathrm{CA}$ have underlying pathologies. Third, a clean capnogram was difficult to obtain during CPR because of numerous issues (device malfunction, leakage/occlusion of ventilation circuit, and ongoing CC effort) $(9,25)$. The accuracy of our results may be related to the analysis of data from an animal model. To avoid the interference of gas oscillation in the ventilation tube caused by $\mathrm{CC}$ with $\mathrm{CO}_{2}$ measurement, ITDs were also used to improve the accuracy of the $\mathrm{V}^{\prime} \mathrm{CO}_{2}$ measurement. However, ITDs are not commonly used in clinical practice, following the current guidelines. Fourth, the results of this animal study may not be suitable for generalization to human patients. Nevertheless, the results show that the PV ratio may have potential applicability in clinical practice.

\section{Conclusions}

In this study, experimental porcine models of primary VF-related CA were used to show that the PV ratio was significantly lower during hyperventilation than under $\mathrm{NV}$ conditions. Additionally, this indicator changed rapidly in response to hyperventilation, reduced immediately after hyperventilation onset, and then slightly increased and stabilized at a low level after approximately $2 \mathrm{~min}$. The $\mathrm{PV}$ ratio showed good performance in discriminating hyperventilation from non-hyperventilation $(\mathrm{AUC}=0.98)$.

\section{Acknowledgments}

We would like to acknowledge Dr. Feng Sun for their constant technical assistance during the experiments.
Funding: This work was supported by the Chinese Academy of Medical Sciences (CAMS) Innovation Fund for Medical Sciences (2017-I2M-1-009); and the Beijing Municipal Science \& Technology Commission (Z181100001718147). The funding was used to purchase experimental animals, experimental drugs, and disposable consumables. The sponsor was not involved in designing the study, collecting and analyzing data, writing the manuscript, or submitting the manuscript for publication.

\section{Footnote}

Reporting Checklist: The authors have completed the ARRIVE reporting checklist. Available at http://dx.doi. org/10.21037/jtd-21-50

Data Sharing Statement: Available at http://dx.doi. org/10.21037/jtd-21-50

Peer Review File: Available at http://dx.doi.org/10.21037/jtd21-50

Conflicts of Interest: All authors have completed the ICMJE uniform disclosure form (available at http://dx.doi. org/10.21037/jtd-21-50). The authors have no conflicts of interest to declare.

Data Citation: Supplementary data associated with this article can be found online at http://dx.doi.org/10.17632/ rn55s98sz8.1

Ethical Statement: The authors are accountable for all aspects of the work in ensuring that questions related to the accuracy or integrity of any part of the work are appropriately investigated and resolved. The Ethics Committee for Animal Experiments at the Peking Union Medical College Hospital approved the experimental protocol (XHDW-2019-023). All animals received humane care in accordance with the "principles of laboratory animal care" formulated by the Ministry of Science and Technology of the People's Republic of China.

Open Access Statement: This is an Open Access article distributed in accordance with the Creative Commons Attribution-NonCommercial-NoDerivs 4.0 International License (CC BY-NC-ND 4.0), which permits the noncommercial replication and distribution of the article with the strict proviso that no changes or edits are made and the 
original work is properly cited (including links to both the formal publication through the relevant DOI and the license). See: https://creativecommons.org/licenses/by-nc-nd/4.0/.

\section{References}

1. Kodali BS, Urman RD. Capnography during cardiopulmonary resuscitation: Current evidence and future directions. J Emerg Trauma Shock 2014;7:332-40.

2. Pantazopoulos C, Xanthos T, Pantazopoulos I, et al. A Review of Carbon Dioxide Monitoring During Adult Cardiopulmonary Resuscitation. Heart Lung Circ 2015;24:1053-61.

3. Silvestri S, Ralls GA, Krauss B, et al. The effectiveness of out-of-hospital use of continuous end-tidal carbon dioxide monitoring on the rate of unrecognized misplaced intubation within a regional emergency medical services system. Ann Emerg Med 2005;45:497-503.

4. Ditchey RV, Winkler JV, Rhodes CA. Relative lack of coronary blood flow during closed-chest resuscitation in dogs. Circulation 1982;66:297-302.

5. Qvigstad E, Kramer-Johansen J, Tømte Ø, et al. Clinical pilot study of different hand positions during manual chest compressions monitored with capnography. Resuscitation 2013;84:1203-7.

6. Pokorná M, Necas E, Kratochvíl J, et al. A sudden increase in partial pressure end-tidal carbon dioxide $(\mathrm{P}(\mathrm{ET}) \mathrm{CO}(2))$ at the moment of return of spontaneous circulation. $\mathrm{J}$ Emerg Med 2010;38:614-21.

7. Soar J, Nolan JP, Böttiger BW, et al. European Resuscitation Council guidelines for resuscitation 2015: Section 3. Adult advanced life support. Resuscitation 2015;95:100-47.

8. Touma O, Davies $M$. The prognostic value of end tidal carbon dioxide during cardiac arrest: a systematic review. Resuscitation 2013;84:1470-9.

9. Herry CL, Townsend D, Green GC, et al. Segmentation and classification of capnograms: application in respiratory variability analysis. Physiol Meas 2014;35:2343-58.

10. Leturiondo M, Ruiz de Gauna S, et al. Influence of chest compression artefact on capnogram-based ventilation detection during out-of-hospital cardiopulmonary resuscitation. Resuscitation 2018;124:63-8.

11. Aufderheide TP, Lurie KG. Death by hyperventilation: a common and life-threatening problem during cardiopulmonary resuscitation. Crit Care Med 2004;32:S345-51.

12. Karlsson T, Stjernström EL, Stjernström H, et al. Central and regional blood flow during hyperventilation. An experimental study in the pig. Acta Anaesthesiol Scand 1994:38:180-6.

13. Aufderheide TP, Sigurdsson G, Pirrallo RG, et al. Hyperventilation-induced hypotension during cardiopulmonary resuscitation. Circulation 2004;109:1960-5.

14. Kreit JW. Volume capnography in the intensive care unit: physiological principles, measurements, and calculations. Ann Am Thorac Soc 2019;16:291-300.

15. Suarez-Sipmann F, Bohm SH, Tusman G. Volumetric capnography: the time has come. Curr Opin Crit Care 2014;20:333-9.

16. Gazmuri RJ, Ayoub IM, Radhakrishnan J, et al. Clinically plausible hyperventilation does not exert adverse hemodynamic effects during CPR but markedly reduces end-tidal PCO2. Resuscitation 2012;83:259-64.

17. Lah K, Križmarić M, Grmec S. The dynamic pattern of end-tidal carbon dioxide during cardiopulmonary resuscitation: difference between asphyxial cardiac arrest and ventricular fibrillation/pulseless ventricular tachycardia cardiac arrest. Crit Care 2011;15:R13.

18. Sheak KR, Wiebe DJ, Leary M, et al. Quantitative relationship between end-tidal carbon dioxide and CPR quality during both in-hospital and out-of-hospital cardiac arrest. Resuscitation 2015;89:149-54.

19. Heradstveit BE, Sunde K, Sunde GA, et al. Factors complicating interpretation of capnography during advanced life support in cardiac arrest--a clinical retrospective study in 575 patients. Resuscitation 2012;83:813-8.

20. Xu J, Yu X, Zhang L, et al. Modified volumetric capnography-derived parameter: A potentially stable indicator in monitoring cardiopulmonary resuscitation efficacy in a porcine model. Resuscitation 2020;150:94-101.

21. Kleinman ME, Brennan EE, Goldberger ZD, et al. Part 5: adult basic life support and cardiopulmonary resuscitation quality: 2015 American Heart Association guidelines update for cardiopulmonary resuscitation and emergency cardiovascular care. Circulation 2015;132:S414-35.

22. Park SO, Shin DH, Baek KJ, et al. A clinical observational study analysing the factors associated with hyperventilation during actual cardiopulmonary resuscitation in the emergency department. Resuscitation 2013;84:298-303.

23. O'Neill JF, Deakin CD. Do we hyperventilate cardiac arrest patients? Resuscitation 2007;73:82-5.

24. Link MS, Berkow LC, Kudenchuk PJ, et al. Part 7: adult 
advanced cardiovascular life support: 2015 American

Heart Association guidelines update for cardiopulmonary resuscitation and emergency cardiovascular care.

Circulation 2015;132:S444-64.

Cite this article as: Zhang L, Liang X, Zhu H, Yin L, Dai J, Liu D, Yu S, Fu Y, Jin K, Xu J, Yu X. New volumetric capnography-derived parameter: a potentially valuable tool for detecting hyperventilation during cardiopulmonary resuscitation in a porcine model. J Thorac Dis 2021;13(6):34673477. doi: 10.21037/jtd-21-50
25. Takla G, Petre JH, Doyle DJ, et al. The problem of artifacts in patient monitor data during surgery: a clinical and methodological review. Anesth Analg 2006;103:1196-204. 